\title{
APPLICATION OF MACHINE LEARNING ALGORITHMS IN STRUCTURAL HEALTH MONITORING RESEARCH
}

\author{
Y. HAMISHEBAHAR*, H.Z. LI and H. GUAN \\ School of Engineering and Built Environment, Griffith University, Gold Coast Campus, QLD 4222, Australia \\ Emails: younes.hamishebahar@griffithuni.edu.au,h.li@griffith.edu.au,h.guan@griffith.edu.au \\ *Corresponding author
}

\begin{abstract}
Engineering structures, including civil infrastructures, have always been susceptible to various kind of damage during their service life. The goal of structural health monitoring is to provide sufficient when the structure condition deteriorates. It has the obvious benefits of preventing disastrous structural collapses and reducing maintenance costs. By and large, structural health monitoring approaches are divided into two classes, model-based, and data-driven approaches. The main challenge in data-driven approaches lies in a large amount of data to be dealt with. As machine learning algorithms are often used to recognize the inherent pattern in data, their applications in data-driven approaches have been gained an increasing attention in recent years. Utilizing machine learning algorithms turns the decision-making step of structural health monitoring into an automated process with minimal human intervention. This paper presents a summary of a literature review concerning different data-driven structural health monitoring approaches combined with machine learning algorithms developed in the last several years. Specifically, this report will review existing applications of machine learning algorithms for the purposes of dimensionality reduction and developing a statistical model for structural health monitoring. The primary aim is to categorise the existing studies in the aforementioned areas in terms of the type of machine learning algorithms. This paper also attempts to identify research gaps to facilitate the formulation of a future study in the aforementioned area.
\end{abstract}

Keywords: Structural Health Monitoring; Data-Driven Approaches; Machine Learning Algorithms;

\section{INTRODUCTION}

Structural and mechanical systems such as bridges, buildings, offshore oil platforms, aircraft, rotating machinery, etc. are key infrastructure assets of a society. Construction defects, aging, and environmental circumstances can adversely affect the life and reliability of these systems. The fact that failure to these systems can cause life-safety and economic consequences highlights that the onset of damage should be detected (Thomson 2013). Damage detection is usually carried out in the context of one or more closely related disciplines including structural health monitoring (SHM), condition monitoring, nondestructive evaluation, health and usage monitoring system, statistical process control, and damage prognosis. SHM is the process of implementing a damage identification strategy for aerospace, civil or mechanical engineering infrastructure. (Farrar and Worden 2012). Note that this paper focuses on the application of machine learning algorithms in SHM of civil infrastructures.

Many portions of our civil infrastructures are approaching or exceeding their initial design life. Because of economic challenges, these civil infrastructures are still being used in spite of 
aging and the associated damage accumulation. Therefore, the ability to monitor the health of these structures is becoming increasingly important (Farrar and Worden 2012).

Generally speaking, damage can be defined as changes introduced into a system that causes a disturbance in its current or future performance. In this study, the definition of damage is narrowed down to the changes to the material and/or geometric properties of structural systems, including changes to the boundary conditions and system connectivity. This can adversely affect the current or future performance of these systems (Sohn et al. 2004).

SHM approaches were first used in the aerospace and mechanical engineering industries for damage diagnosis and were then introduced to offshore platform structures in the 1970s (Vandiver 1975). In the following three decades, the number of studies and the application of SHM to civil engineering structures, such as bridges, dams, etc., have been increased (Zhou et al. 2013). So far, numerous SHM approaches and techniques have been proposed by different researchers. As SHM is a multidisciplinary research, the number of studies in this area has become more significant. That is also the reason why literature review papers with different perspectives in this area have been published. References (Ciang et al. 2008; Im et al. 2011; Mitra \& Gopalakrishnan 2016; Montalvao et al. 2006; Sohn et al. 2004) are examples that provide literature reviews in the area of SHM with different applications and perspectives.

In order to make an informed decision on damage detection, it is required to compare the current properties of the structure with a baseline (Worden et al. 2007). In general, with respect to the baseline data, SHM approaches are categorized into two classes: model-based and datadriven approaches. Model-based approaches require establishing a physical model of the structure, usually by the finite element method. Identifying the occurrence, location, and severity of the damage in model-based approaches can be achieved by the solution of an inverse problem. Friswell (Friswell 2007) prepared an overview of some common methods for solving the inverse problem.

In data-driven approaches, a model is also established, but here the model is usually a statistical representation of the system. Different algorithms have been developed over the years for data-driven approaches. These algorithms are mainly drawn from the machine learning discipline. Machine learning is the scientific study of algorithms and statistical models that computer systems use in order to perform a specific task effectively without using explicit instructions, relying on patterns and inference instead. By and large, the machine learning algorithms come from two main classes: supervised and unsupervised learning. In supervised learning, the algorithm builds a mathematical model from a set of data that contains both the inputs and the desired outputs. On the other hand, in unsupervised learning, the algorithm builds a mathematical model from a set of data which contains only inputs and no desired outputs. Unsupervised learning algorithms are used to find structure in data (Bishop 2006).

Farrar et al. (Farrar et al. 1999) expressed that a data-driven approach of SHM is composed of four portions: 1. Operational evaluation. 2. Data acquisition and cleansing. 3. Feature extraction and data compression. 4. Statistical model development. Machine learning algorithms are mainly used in the last step to develop a statistical model and in some cases, they are used in the third step for dimensionality reduction.

In this paper, different applications of machine learning algorithms for both dimensionality reduction and for developing a statistical model are investigated. The goal is to conduct a review of the application of machine learning algorithms in the area of SHM to identify a research gap to facilitate the formulation of a future study in this area.

The organization of this paper is as follows. Section 2 deals with the task of dimensionality reduction using machine learning algorithms. Section 3 narrates the application of both supervised and unsupervised machine learning algorithms for developing a statistical model which is the main application in the SHM area. Section 4 highlights a brief conclusion of this study. 


\section{FEATURE EXTRACTION AND DIMENSIONALITY REDUCTION}

The third step of developing a data-driven SHM system, feature extraction and data compression, has a profound influence on the performance of a SHM system. Feature extraction is defined as turning the raw signals into low dimensional vectors which are sensitive to the existence of damage. It is usually necessary to convert measured data into features. This has the obvious benefit of retaining the necessary information and discarding the redundant information from raw signals (Worden and Manson 2007). Different methods have been proposed for extracting damage sensitive features. In some cases, machine learning algorithms are used to reduce the dimension of data. There are several machine learning algorithms that are used to discover the better representation of the inputs and to reduce the dimension of input data (Bengio et al. 2013). Among these algorithms, a classic example which is popular in the area of SHM would be principal component analysis (PCA).

PCA has various applications in data mining and in analyzing fields such as dimensionality reduction, data visualization, outlier detection, and correlation analysis. In the area of SHM, PCA has been used for dimensionality reduction and visualization. PCA was utilized for both dimensionality reduction and for data visualization in a proposed SHM approach to detect damage in experimental and numerical structures (de Lautour and Omenzetter 2010). They also used another technique, i.e., selection of subsets of auto-regressive (AR) coefficients for dimensionality reduction. Their results showed that for larger feature dimensions, PCA outperforms the selection of subsets of AR coefficients. PCA along with continuous Daubechies wavelet is used for feature extraction (Kesavan and Kiremidjian 2012). In this study, damage sensitive features are obtained by extracting the minimum variance obtained by the PCA of wavelet energies.

Implementation of PCA is simple and effective for dealing with linear cases. However, for complicated cases, especially nonlinear cases, PCA is unsuccessful as it is linear by nature. As a modification of PCA, kernel principal component analysis (KPCA) has been used in recent years for dealing with nonlinear problems. In the area of fault diagnosis, Yuqing et al. (2015) utilized a data-dependent KPCA technique to transform original high-dimensional data into low-dimensional manifold feature space with the intrinsic dimensionality. They compared the results with the approach including PCA instead of KPCA. It was observed that the KPCAbased method outperforms the PCA-based one. Another comparison between PCA and KPCA for dimensionality reduction in the area of damage detection was investigated by Santos et al. (Santos et al. 2019). They expressed that for the feature vector, the fact that there is a large number of features and a small number of measurements highlights the need for dimensionality reduction. Their results showed that KPCA can maintain a monotonic relationship between the progressive level of damage and the amplitude of damage indicator. In contrast, the PCA failed to achieve the monotonic relationship and exhibited many false-negative indications of damage for both scenarios.

\section{STATISTICAL MODEL DEVELOPMENT}

In the last step of developing a data-driven SHM system, the goal is to perform a statistical comparison with the baseline. The decision on using the type of learning (i.e. supervised and unsupervised) is made based on the availability of data from damaged states of the structure. If the data from the damaged state of the structure is available (e.g. extracted from either an experimental or numerical model), the learning type would be supervised. In contrast, in the context of SHM, the unsupervised learning requires data only from the undamaged state of the structure (i.e. the input data). It is concerned with determining the underlying structure in data from undamaged states of the structure (Worden and Manson 2007). 


\subsection{Supervised Learning}

Different supervised algorithms have been proposed so far. However, artificial neural networks (ANNs), support vector machine (SVM), and k-nearest neighbors $(\mathrm{kNN})$ are more popular algorithms used by researchers. Two supervised pattern recognition techniques based on nearest neighbors classification (NNC) and learning vector quantization (LVQ) were used to classify damage based on analysis of time series model coefficients (de Lautour and Omenzetter 2010). Acceleration time histories acquired from the book-shelf structure proposed by the Los Alamos laboratory and a benchmark structure proposed by the American Society of Civil Engineers (ASCE) were used to validate the method. Another application of $\mathrm{kNN}$ for damage detection of the benchmark ASCE structure was done under a combination with wavelet packet decomposition (WPD) as the feature extraction method (Zhou et al. 2014). The authors compared the aforementioned combination with a new combination including feature selection via random forest variable importance analysis and random forest recursive feature elimination. They concluded that the accuracy obtained from the new feature sequences is much higher than that from the original WPD feature sequence.

Support vector machine (SVM) is another popular classification algorithm. It was used to perform a supervised multi-class classification to detect damage in a numerical three-story structure equipped with MR damper (Chong et al. 2014). In the case of utilizing the SVM algorithm, an important step is choosing the kernel function. In order to improve the performance of classification, different kernel functions have been introduced by researchers. A new combinational kernel function, so-called "Thin plate spline Littlewood-Paley wavelet (TPSLPW) kernel function", was introduced by Ghiasi et al. (2016). They detected and located damage in two numerical benchmark studies using SVM which utilizes PSLPW as the kernel function. Their results showed that the performance of the least square SVM algorithm is improved by utilizing the proposed kernel function.

Artificial Neural Networks (ANNs) have been applied in several branches of science and engineering for years. Researchers have conducted different studies in the area of SHM by utilizing ANNs. Their ability to simulate the behavior of systems with high nonlinearity, flexibility, and robustness are some of the advantages related to using the ANNs (Bani-Hani et al. 1999). In another study, ANNs were used to detect damage occurrence, location, and severity in a simply supported steel I-beam (Tan et al. 2017). They used the ANNs incorporating a modal strain energy-based damage index as the input layer to quantify damage severities under multiple damage scenarios. Their findings demonstrated the accuracy and efficiency of their proposed method in performance evaluation and failure prevention of steel beams. The applicability of using ANNs in a supervised, under noise contamination setting, and availability of partially modal properties as input was investigated by Chang et al. (2018). Their results demonstrated that only slight changes are observed on predicting damage index, level, and location when the data are noise-contaminated. So, their proposed system can reliably be used under noise interference.

In addition to the well-known supervised algorithms, there are research studies in which a new introduced supervised algorithm is utilized to see whether they are useful in the area of SHM. A new supervised algorithm titled "neural dynamic classification (NDC)" was utilized to detect damage in a high-rise building (Rafiei and Adeli 2017). In order to perform a supervised classification, NDC was used and compared with $\mathrm{kNN}$, probabilistic neural network (PNN), and enhanced PNN (EPNN). Their results showed that the NDC is more accurate than $\mathrm{kNN}$ and PNN for all cases defined in their study and more accurate than EPNN in most cases.

\subsection{Unsupervised Learning}

In the area of SHM, by relying on data from undamaged states only, the approaches seek to flag data acquired from a damaged state of the structure as anomaly or outlier (Long and 
Büyüköztürk 2017). In data mining, anomaly detection (also outlier detection) is the identification of rare items, events or observations which raise suspicions by differing significantly from the majority of the data (Zimek and Schubert 2017). Different approaches have been considered for detecting anomalies so far.

Several studies have used measures of distance for multivariate statistics outlier detection. Mahalanobis squared distance (MSD) is one of the popular measures of distance to perform an anomaly detection approach. One of the earliest studies with an unsupervised anomaly detection manner was done by Worden et al. (2000). They proposed a method of damage detection adapting anomaly detection based on MSD. They expressed that in choosing the MSD, there is an implicit assumption that the normal condition datasets have Gaussian statistics. MSD was utilized in another study to compete with three other unsupervised algorithms to detect damage in a benchmark base-excited laboratory frame structure proposed by Los Alamos (Figueiredo et al. 2011). They concluded that the MSD-based algorithm is revealed to be the best data normalization approach in terms of the classification performance, the reduced computational efforts, and the fact that no assumptions are required regarding its architecture. MSD is also compared with a novel distance method called as partition-based Kullback-Leibler divergence (PKLD) proposed by Entezami et al. (2018). They used PKLD to measure the discrepancy between the AR model residuals of undamaged and damaged conditions for damage detection and localization. They stated that the comparative analysis of the performances of the PKLD and MSD methods demonstrates that both of them are able to detect and locate damage. However, the proposed PKLD method outperforms MSD in terms of false-positive and falsenegative indication of damage.

Another way of implementing an anomaly detection approach is to develop a statistical model on normal data. Then, an investigation is done to see whether the newly acquired data come from the same source or distribution. If the new test data come from different source or distributions, they might be flagged as data related to a possible damaged state (Worden and Manson 2007). One of the popular algorithms in the aforementioned approach is One-Class SVM (OCSVM). Das et al. (2010) presented an approach to characterize and classify different damaged states in composite laminates using OCSVM. They compared its performance with the anomaly detection version of $\mathrm{kNN}$ and suggested that OCSVM with appropriate preprocessing techniques can often achieve better accuracy than $\mathrm{kNN}$ in detecting and classifying anomalies. OCSVM along with three other kernel-based algorithms were utilized to form a comparative study focusing on damage detection using anomaly detection approach. It was observed that in general, kernel-based algorithms for anomaly detection outperform four other alternative algorithms which dealing with anomaly detection without kernel functions (Santos et al. 2016). A comprehensive application of OCSVM and least square OCSVM for detecting and localizing damage in a three-story steel-frame experimental structure can be found in (Long and Büyüköztürk 2017). They introduced a novel variant of the aforementioned algorithms, so-called "early stopping one-class kernel least square" which is trained through a faster and simpler procedure to implement iterative procedure than other kernel classification methods while retaining the same advantages.

An anomaly detection approach can also be performed using cluster analysis. Cluster analysis is a type of unsupervised learning that automatically forms clusters of similar things (Harrington 2012). In this approach, after clustering the input data, any data point not belonging to any cluster can be flagged as an outlier. The situation goes on for any new test data, as well. Gaussian mixture models (GMMs) are frequently used as clustering algorithms in pattern recognition. A comprehensive application of GMMs combined with MSD was done to investigate the occurrence and extent of damage in the benchmark ASCE structure (Nair et al. 2006). Damage detection was done using gap static which ascertains the optimal number of mixtures in a particular dataset. They used MSD as an indicator of damage extent. They also 
investigated the performance of their proposed approach when the data are noise-contaminated. They showed that with applying noise, only the smallest damage imposed on the structure is not detected and for the rest of the damage cases, results are promising. Detection of damage occurrence using a clustering algorithm and damage extent using a distance measure was done again with a combination of K-means and Euclidean as clustering algorithm and distance measure, respectively (Kesavan and Kiremidjian 2012). K-means algorithm was used to estimate the mixture centers in the dataset and the gap static was utilized to determine the optimal number of clusters. The authors expressed that the reason for choosing this combination over the previous one (i.e. GMMs and MSD) is that the new combination provides greater computational efficiency. A new clustering algorithm, titled "density peaks-based fast clustering" was modified to an unsupervised machine learning method by adding training and testing process to improve damage detection and localization of multi-story and multi-bay structures (Cha and Wang 2018). The authors also compared its performance with the wellknown anomaly detection OCSVM. They showed that their proposed algorithm outperforms OCSVM. Santos et al. (2019) proposed a new approach for feature extraction including transmissibilities and KPCA (Santos et al. 2019). The theory provided in their study was extended to classify damaged and undamaged cases via a density-based clustering procedure known as mean shift clustering (MSC). Their results showed that their proposed approach can well minimize false-positive and false-negative errors.

\section{CONCLUSION}

In this paper, a brief review of the application of machine learning algorithms in the area of SHM for dimensionality reduction and statistical model development was conducted. Statistical model development was categorized into two classes of supervised and unsupervised approaches. Although different SHM studies have been done with a supervised setting, the limitation of obtaining the data from damaged states of the structure which requires establishing either a finite element or experimental model is a disadvantage for the supervised setting. The unsupervised setting is more compatible with the type of data existed for real structures and the authors are going to focus on SHM approaches with this learning manner. As it was observed, mainly, the novelty related to the studies in this area comes from introducing a new combination of algorithms for both feature extraction and statistical model development steps. Both of the aforementioned steps deeply affect the performance of a SHM system. The authors are going to link the feature extraction step to the statistical model development step. In other words, the development of a new feature fusion method based on the mechanism of anomaly detection algorithms is going to be investigated. The proposed approach is going to fuse the promising feature extraction methods in a way guaranteeing that the best possible accuracy will be obtained in the next step. This makes the SHM process more automated and in turn, the human intervention can be much less required.

\section{REFERENCES}

Bani-Hani, K., Ghaboussi, J. and Schneider, S. P. (1999) 'Experimental study of identification and control of structures using neural network. Part 1: identification', Earthquake Engineering \& Structural Dynamics. John Wiley \& Sons, Ltd, 28(9), pp. 995-1018. doi: 10.1002/(SICI)1096-9845(199909)28:9<995::AID-EQE851>3.0.CO;2-8.

Bengio, Y., Courville, A. and Vincent, P. (2013) 'Representation learning: A review and new perspectives', IEEE transactions on pattern analysis and machine intelligence. IEEE, 35(8), pp. 1798-1828.

Bishop, C. M. (2006) Pattern recognition and machine learning. springer. 
Cha, Y.-J. and Wang, Z. (2018) 'Unsupervised novelty detection-based structural damage localization using a density peaks-based fast clustering algorithm', Structural Health Monitoring. SAGE Publications Sage UK: London, England, 17(2), pp. 313-324.

Chang, C.-M., Lin, T.-K. and Chang, C.-W. (2018) 'Applications of neural network models for structural health monitoring based on derived modal properties', Measurement. Elsevier, 129, pp. 457-470.

Chong, J. W., Kim, Y. and Chon, K. H. (2014) 'Nonlinear multiclass support vector machinebased health monitoring system for buildings employing magnetorheological dampers', Journal of Intelligent Material Systems and Structures, 25(12), pp. 1456-1468. doi: 10.1177/1045389X13507343.

Ciang, C. C., Lee, J.-R. and Bang, H.-J. (2008) 'Structural health monitoring for a wind turbine system: a review of damage detection methods', Measurement science and technology. IOP publishing, 19(12), p. 122001.

Das, S., Chattopadhyay, A. and Srivastava, A. N. (2010) 'Classifying induced damage in composite plates using one-class support vector machines', AIAA Journal, 48(4), pp. 705-718. doi: 10.2514/1.37282.

Entezami, A., Shariatmadar, H. and Karamodin, A. (2018) 'Data-driven damage diagnosis under environmental and operational variability by novel statistical pattern recognition methods', Structural Health Monitoring. SAGE Publications Sage UK: London, England, p. 1475921718800306.

Farrar, C. R. et al. (1999) 'A statistical pattern recognition paradigm for vibration-based structural health monitoring', Structural Health Monitoring. Citeseer, 2000, pp. 764-773.

Farrar, C. R. and Worden, K. (2012) Structural health monitoring: a machine learning perspective. John Wiley \& Sons.

Figueiredo, E. et al. (2011) 'Machine learning algorithms for damage detection under operational and environmental variability', Structural Health Monitoring. Sage Publications Sage UK: London, England, 10(6), pp. 559-572.

Friswell, M. I. (2007) 'Damage identification using inverse methods', Philosophical Transactions of the Royal Society of London A: Mathematical, Physical and Engineering Sciences. The Royal Society, 365(1851), pp. 393-410.

Ghiasi, R., Torkzadeh, P. and Noori, M. (2016) 'A machine-learning approach for structural damage detection using least square support vector machine based on a new combinational kernel function', Structural Health Monitoring: An International Journal, 15(3), pp. 302-316. doi: 10.1177/1475921716639587.

Harrington, P. (2012) Machine learning in action. Manning Publications Co.

Im, S. B., Hurlebaus, S. and Kang, Y. J. (2011) 'Summary review of GPS technology for structural health monitoring', Journal of Structural Engineering. American Society of Civil Engineers, 139(10), pp. 1653-1664.

Kesavan, K. N. and Kiremidjian, A. S. (2012) 'A wavelet-based damage diagnosis algorithm using principal component analysis', Structural Control and Health Monitoring. Wiley Online Library, 19(8), pp. 672-685.

de Lautour, O. R. and Omenzetter, P. (2010) 'Nearest neighbor and learning vector quantization classification for damage detection using time series analysis', Structural Control and Health Monitoring. Wiley Online Library, 17(6), pp. 614-631.

Long, J. and Büyüköztürk, O. (2017) 'Decentralised one-class kernel classification-based damage detection and localisation', Structural Control and Health Monitoring. Wiley Online Library, 24(6).

Mitra, M. and Gopalakrishnan, S. (2016) 'Guided wave based structural health monitoring: A review’, Smart Materials and Structures. IOP Publishing, 25(5), p. 53001. 
Montalvao, D., Maia, N. M. M. and Ribeiro, A. M. R. (2006) 'A review of vibration-based structural health monitoring with special emphasis on composite materials', Shock and vibration digest. Bournemouth University, Fern Barrow, Poole, Dorset, BH12 5BB, UK, 38(4), pp. 295-324.

Nair, K. K., Kiremidjian, A. S. and Law, K. H. (2006) 'Time series-based damage detection and localization algorithm with application to the ASCE benchmark structure', Journal of Sound and Vibration. Elsevier, 291(1-2), pp. 349-368.

Rafiei, M. H. and Adeli, H. (2017) 'A novel machine learning-based algorithm to detect damage in high-rise building structures', The Structural Design of Tall and Special Buildings. Wiley Online Library, 26(18), p. e1400.

Santos, A. et al. (2016) 'Machine learning algorithms for damage detection: Kernel-based approaches', Journal of Sound and Vibration. Elsevier, 363, pp. 584-599.

Santos, A. et al. (2019) 'Output-only structural damage detection based on transmissibility measurements and kernel principal component analysis', Journal of Communication and Information Systems, 34(1), pp. 64-75.

Sohn, H., Farrar, C. R. and Hemez, F. M. (no date) 'A review of structural health monitoring literature: 1996-2001'.

Tan, Z. X. et al. (2017) 'Detecting damage in steel beams using modal strain energy based damage index and Artificial Neural Network', Engineering Failure Analysis. Elsevier, 79, pp. 253-262.

Thomson, D. J. (2013) 'The economic case for service life extension of structures using structural health monitoring based on the delayed cost of borrowing', Journal of Civil Structural Health Monitoring, 3(4), pp. 335-340. doi: 10.1007/s 13349-013-0057-0.

Vandiver, J. K. (1975) 'Detection of structural failure on fixed platforms by measurement of dynamic response', in Offshore Technology Conference. Offshore Technology Conference.

Worden, K. et al. (2007) 'The fundamental axioms of structural health monitoring', in Proceedings of the Royal Society of London A: Mathematical, Physical and Engineering Sciences. The Royal Society, pp. 1639-1664.

Worden, K. and Manson, G. (2007) 'The application of machine learning to structural health monitoring', Philosophical Transactions of the Royal Society of London A: Mathematical, Physical and Engineering Sciences. The Royal Society, 365(1851), pp. 515-537.

Worden, K., Manson, G. and Fieller, N. R. J. (2000) 'Damage detection using outlier analysis', Journal of Sound and Vibration. Elsevier, 229(3), pp. 647-667.

Yuqing, Z. et al. (2015) 'NC machine tools fault diagnosis based on kernel PCA and-nearest neighbor using vibration signals', Shock and Vibration. Hindawi, 2015.

Zhou, L. et al. (2013) 'Review of benchmark studies and guidelines for structural health monitoring', Advances in Structural Engineering. SAGE Publications Sage UK: London, England, 16(7), pp. 1187-1206.

Zhou, Qifeng et al. (2014) 'Structure damage detection based on random forest recursive feature elimination', Mechanical Systems and Signal Processing. Elsevier, 46(1), pp. 82-90.

Zimek, A. and Schubert, E. (2017) 'Outlier Detection BT - Encyclopedia of Database Systems', in Liu, L. and Özsu, M. T. (eds). New York, NY: Springer New York, pp. 1-5. doi: 10.1007/978-1-4899-7993-3_80719-1. 